\title{
Consensus statement: improvement in care and outcomes for patients with ST-elevation myocardial infarction with timely reperfusion therapy: a pharmaco-invasive approach
}

\author{
Arup Dasbiswas $^{1 *}$, Abhijeet Shelke ${ }^{2}$, Anurag Singhal ${ }^{3}$, Bhupesh Shah ${ }^{4}$
}

\author{
${ }^{1}$ Interventional Cardiology, Arup Das Biswas Clinic, Kolkata, West Bengal, India \\ ${ }^{2}$ Interventional Cardiology and Electrophysiology, Krishna Institute of Medical Sciences, Karad, Maharashtra, India \\ ${ }^{3}$ Interventional Cardiology, Ajanta Heart Care and Diagnostic Centre, Ghaziabad, Uttar Pradesh, India \\ ${ }^{4}$ Interventional Cardiology, HCG Hospital, Ahmedabad, Gujarat, India
}

Received: 04 August 2020

Accepted: 08 September 2020

\section{*Correspondence:}

Dr. Arup Dasbiswas,

E-mail: arup.dasbiwas@gmail.com

Copyright: (c) the author(s), publisher and licensee Medip Academy. This is an open-access article distributed under the terms of the Creative Commons Attribution Non-Commercial License, which permits unrestricted non-commercial use, distribution, and reproduction in any medium, provided the original work is properly cited.

\begin{abstract}
The prevalence of cardiovascular diseases continues to increase in India, with a rapid epidemiological transition. Timely management of ST-elevation myocardial infarction (STEMI)is the key to decrease mortality and morbidity rates. In India, patients most often do not receive optimal therapy owing to a number of factors such as socioeconomic constraints, lack of knowledge, geographical restrictions etc, leading to suboptimal outcomes. The timely delivery of optimal reperfusion strategy for STEMI patients is immensely challenging for physicians, due to several reasons. Primary percutaneous coronary Intervention (PCI) is still a global gold standard for the treatment of STEMI. Delayed case presentation is a significant challenge, especially in Indian tier towns/cities, because of prolonged transportation time or traffic delays with regards to reaching a PCI facility. Keeping in mind the accessibility to PCI facility because of geographical locations as well as affordability concerns especially in smaller Indian towns, pharmaco-invasive approach is almost as effective and a practical approach for reduction of morbidity and mortality rates. Timely establishment of reperfusion therapy is critical for improving myocardial salvage in patients with STEMI. There is an urgent need for developing strategies for efficient STEMI management, such as timely delivery of reperfusion therapy; reinforcement of health system and professionals, implementation of guideline-based interventions, streamlined insurance policy and greater emphasis on patient awareness for detection of symptoms. In addition to the timely reperfusion therapy and other standard care drugs, statins and potassium channel activators have demonstrated clinically significant improvements in the outcome of STEMI management.
\end{abstract}

Keywords: Acute coronary syndrome, Pharmaco-invasive therapy, ST-elevation myocardial infarction, Cardiac rehabilitation

\section{INTRODUCTION}

Cardiovascular diseases (CVD) are reported to be a leading cause of mortality in Indian population. ${ }^{1}$ According to global burden of disease reports, India (272 per 100,000 population) presents higher age standardized CVD death rates than the global population (235 per
100,000 population). ${ }^{2}$ Acute coronary syndrome constituting ST-elevation myocardial infarction (STEMI), $\mathrm{N}$-STEMI and unstable angina conditions is responsible for one-third of total CVD deaths. ${ }^{3}$ STEMI is defined as the characteristic clinical symptom of myocardial ischemia (MI), with persistent electrocardiographic STE, and the subsequent release of biomarkers of myocardial 
necrosis. ${ }^{4}$ Approximately 3 million STEMI cases are reported in India every year, and only $20 \%$ of the patients can afford proper treatment and care. ${ }^{2}$ There are a number of factors that are responsible for delay in STEMI management and these need to be addressed carefully at each step.

The aim of this article was to collate expert experience and opinions on the significance of pharmaco-invasive therapy in STEMI patients when primary PCI cannot be performed in timely manner, in tier $1 / 2$ cities of India. The present paper highlights the important points put forth in an expert meeting, held in eight cities of India, in order to improve care and outcomes in patients presenting with STEMI.

\section{METHODOLGY}

A series of expert meetings involving specialists like cardiologists and physicians were conducted in eight cities of India (Figure 1). These experts shared their experience on the importance of PCI therapy during STEMI management and the significance of pharmacoinvasive therapy in STEMI patients when primary PCI cannot be performed in timely manner. Individual expert meeting opinions were collected and finally their consensus was compiled into one document.

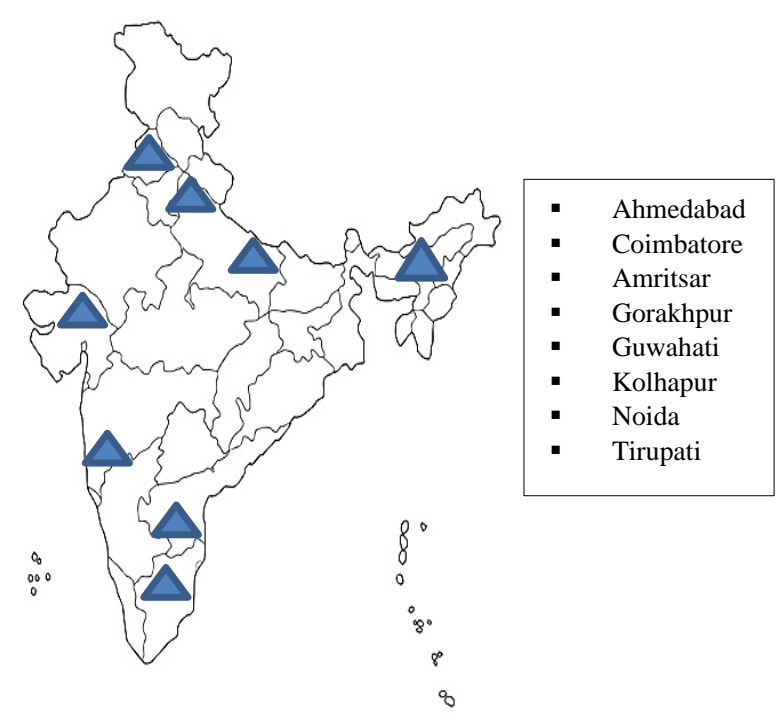

Figure 2: Locations of the expert meetings in 8 Indian tier cities.

\section{STEMI MANAGEMENT}

Percutaneous coronary intervention (PCI) is the first choice for management of STEMI patients, as it has been reported to result in a higher percentage of lasting and complete reperfusion. Primary PCI intervention restores normal flow angiographically, in the previously occluded artery, in more than $90 \%$ of patients. ${ }^{5}$ In STEMI setting, PCI is the preferred intervention- provided it can be initiated as per recommended timelines in an appropriate manner.6 The door to balloon time for PCI should be achieved in patients presenting within 90 minutes or $>120$ minutes respectively. 5,6 The expert consensus meeting also highlighted the importance of timely intervention of PCI in patients presenting with STEMI.

Timely PCI Intervention PRAMI (preventive angioplasty in acute myocardial infarction) trial, demonstrated a significant decrease in incidence of lower ischemic outcomes in STEMI patients undergoing complete revascularization at time of primary PCI, including nonculprit vessel PCI. $^{7}$ TRIANA trial reported that primary PCI is an appropriate intervention for STEMI cases even for the elderly population. ${ }^{8}$ CvLPRIT (complete versus lesion-only primary pci trial)trial demonstrated a significant reduction in major adverse cardiac events (MACE) endpoints in patients who underwent multivessel PCI. $^{9}$ Based on the available clinical evidences and landmark trails, recent guidelines also suggest timely intervention of PCI as an appropriate reperfusion therapy in patients presenting with myocardial infarction. ${ }^{10}$

\section{Hurdles for STEMI Management in Indian towns}

However, in a developing country like India, timely PCI continues to be a challenge owing to the following factors: ${ }^{2,5,6,10}$

1) Delay in seeking medical attention or calling an ambulance 2) lack of awareness about the disease and its early signs and symptoms 3) dearth of PCI centers in remote areas 4) lack of $24 \times 7$ availability of PCI centers in metro cities as well as in smaller towns 5) poorly trained paramedics and technicians 6) transportation difficulty and traffic delays: Timely transfer to PCI centers remains a huge challenge considering the dearth of emergency ambulance services and poor state of road infrastructure 7) delays within a care center due to factors such as availability of PCI centre during busy hours, arrangement of finances, obtaining consent or availability of round the clock workforce 8) arranging finances on an emergency basis and lack of health insurance policies.

This expert committee also put forth the need gaps in timely management of STEMI.

"In Indian scenario, frequency of primary PCI intervention in hospitals is very low due to the challenges faced due to lack of transfer facility, financial constraints, unavailability of cardiologists around the clock and difficulty in obtaining patient/relative consent."

The "Create" and "Kerala" ACS registries stated that in Indian population there is a significant delay in patient presentation and initiation of timely reperfusion therapy. ${ }^{11,12}$ Create ACS registry study was conducted in 89 centers from 10 regions and 50 cities in India, aimed to improve treatment outcomes in ACS patients. It demonstrated that in Indian scenario, most patients were 
from lower middle $(52.5 \%)$ classes and median time taken from symptoms to hospital was 360 minutes, with 50 minutes from hospital to thrombolysis. It included majority of patients from cities in India with PPCI facilities available, median time from symptom onset to reach hospital being 300 minutes. ${ }^{12}$ In addition to this, Kerala ACS registry evaluated data from 25,748 ACS admissions in 125 Kerala hospitals to address treatment and health system gaps during intervention. Data registry reported that thrombolytics was used in $41 \%$ STEMI, $19 \%$ non-STEMI and $11 \%$ unstable angina cases. PCI (Percutaneous coronary intervention rates) rates were found marginally higher in STEMI admissions. The available data demonstrated that ACS management can be improved by focusing on factors which help in reducing the symptom-to-door time or door-to-needle time. ${ }^{11}$ Reduction in delays in access to hospital and provision of affordable treatments could significantly reduce morbidity and mortality rates.

In such a scenario, pharmaco-invasive (PI) therapy has been reported to play a significant therapeutic role. PI therapy is a strategy consisting of early fibrinolysis followed by transferring the patient to a PCI-capable center either for immediate PCI for patients with failed thrombolysis, or for non-urgent coronary angiography to regulate the necessity for additional revascularization within 3 to 24 hours. ${ }^{13}$ Several clinical trials have also supported this approach to be adopted for patients with acute STEMI, where PCI cannot be performed. ${ }^{14,15}$

The expert group members were of the same opinion for adoption of pharmaco-invasive therapy in Indian scenario:

"Keeping in view the Indian scenario, pharmaco-invasive approach is a more practical and suitable option in patients who present after long time following symptom onset."

PCI is the gold standard of therapy although in order to further reduce rehospitalization and mortality rates in patients with STEMI where PCI is unapproachable, improvements or alternative management is required. It is important to address factors which are responsible to improve survival rates in patients presenting with STEMI, to decrease incidence of downstream rehospitalization, secondary $\mathrm{MI}$, myocardial injury or even heart failure. ${ }^{16-18}$ Another significant reason behind secondary attack in post-MI patients is lack of adherence to a medication regimen. ${ }^{19}$ PCI rates are also very low because of accessibility and cost issues and that is why pharmaco-invasive therapy is the way ahead for patients with acute coronary syndrome..$^{6,10,16,17}$ Taking into consideration the presence of various logistic barriers in timely PCI management in Indian scenario, Sharma (2018), reaffirmed that pharmaco-invasive approach is a safe alternative for STEMI patients presenting at non-PCI centers in India. ${ }^{20}$ Several other clinical trials such as Caress-In-Ami, Transfer-Ami, West Study, Stream, Step
Ami have reported the potential benefits of pharmacoinvasive strategy. ${ }^{5}$

\section{Pharmaco-invasive therapy}

Pharmaco-invasive (PI) therapy has tremendous significance in Indian scenario. It involves initially administering fibrinolytic agents and then systematically performing an angiography within 3-24 hours, after start of fibrinolytic therapy regardless of whether fibrinolysis results in successful reperfusion or not. ${ }^{21}$ The use of antiplatelets, $\beta$-blockers, statins and angiotensin converting enzyme inhibitors (ACEIs)/angiotensin receptor blockers (ARBs in ACEI intolerant patients) in STEMI patients reduced the clinical risk of mortality and MACE events. $^{2}$ It has also been reported, that an impressive number of patients (5-10\%) presenting with ACS, have atrial fibrillation and use oral anticoagulants $(\mathrm{OAC}){ }^{22}$

Although primary PCI facility is available in most of the Indian metro and urban cities, it is limited by several factors such as cost of procedure, traffic congestion, lack of $24 \times 7$ availability to STEMI facilities and increased footfall in the PCI centres.23 Suburban hospitals may also be plagued with limited facility in PCI cathlabs. Thus, the PI approach is of utmost importance in Indian scenario due to unavailability of PCI facilities in small cities, towns and villages (Figure 2). ${ }^{21,24}$

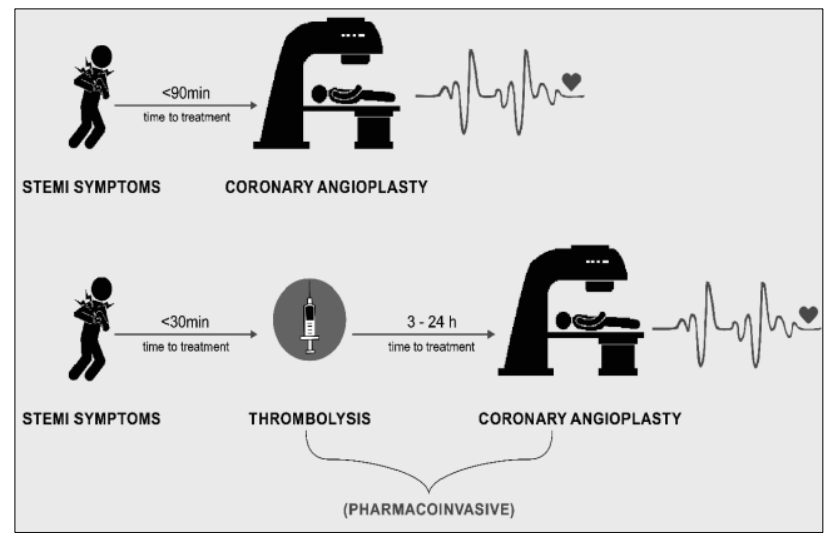

Figure 2: Pharmaco-invasive approach of STEMI care.

\section{Thrombolytic agents in PI approach for optimizing STEMI management}

Thrombolytic therapy for acute myocardial infarction (AMI) was incorporated into the armamentarium of clinicians over 2 decades ago. Thrombolytic therapy recanalizes the thrombotic occlusion related with STEMI and the restoration of coronary flow reduces infarct size. It also improves myocardial function and survival over short and long-term. ${ }^{26}$ Thrombolytics plays a vital role in cardio-vascular disease management. An effective treatment should concentrate on rapid clot dissolution, reduction of tissue damage and boosting survival rates. ${ }^{27}$ 
The evolution of the thrombolytics over the last two decades has seen a significant transition from first generation (streptokinase, urokinase) to fibrin-specific, nonantigenic, second generation (alteplase) and third generation thrombolytics (reteplase, tenecteplase). This evolution is associated with longer half-lives, resistance to plasminogen activator inhibitor-1, better 90 minutes patency rates and TIMI flow grade 3 . In a given health care setting, the choice of thrombolytic agent should consider factors such as mortality risk, intracranial hemorrhage, age, timing of thrombolytic treatment and cost effectiveness. ${ }^{26}$

Indian consensus meeting (2013) recommends a time guided fibrinolytic therapy either with reteplase, tenecteplase, alteplase or streptokinase along with adjunctive PI therapy when PCI cannot be done in given timelines. $^{21,28}$ These have demonstrated superior clinical efficacy, safety and tolerability along with rapid reperfusion, effective clot lysis activity, easy administration and minimal risk of mishandling or dose errors over other fibrinolytic agents. ${ }^{21,29}$ It has also reported $50 \%$ lowering of ST-segment in greater number of patients at 90 minutes post dosing and improves long term survival. ${ }^{29}$

The third-generation fibrinolytic agents e.g. reteplase has increased fibrin specificity, increased resistance to inhibition by plasminogen activators and longer half-life. Reteplase is a fibrin-specific recombinant plasminogen activator derived from t-PA. The benefits of reteplase (3rd generation) are higher than other fibrinolytic agents such as its feasibility to administer a double dosing regimen resulting in rapid reperfusion. Dose adjustment on the basis of body weight is not required in reteplase therapy. It has proven superiority in patency rates compared to first/second generation lytic agents. It actively penetrates the clot rather than accumulating on the top, thus leading to effective clot lysis. Comparatively greater ease of administration and lesser chances of dosing errors are seen as infusion is not required during reteplase administration. . $^{21,26,27}$

Table 1: Comparison of fibrinolytic agents. ${ }^{26}$

\begin{tabular}{|c|c|c|c|c|}
\hline Parameters & Streptokinase & Alteplase & Reteplase & Tenecteplase \\
\hline Dose & $\begin{array}{l}1.5 \mathrm{MU} \text { in } 30- \\
60 \mathrm{~min}\end{array}$ & $\begin{array}{l}\text { Up to } 100 \mathrm{mg} \text { in } 90 \mathrm{~min} \\
\text { (based on weight) }\end{array}$ & $\begin{array}{l}10 \mathrm{U} \times 2(30 \mathrm{~min} \text { apart }) \\
\text { each over } 2 \mathrm{~min}\end{array}$ & $\begin{array}{l}30-50 \mathrm{mg} \text { based } \\
\text { on Weight }\end{array}$ \\
\hline Bolus administration & No & No & Yes & Yes \\
\hline $\begin{array}{l}\text { Antigenic allergic } \\
\text { reactions }\end{array}$ & Yes & No & No & No \\
\hline $\begin{array}{l}\text { Systemic fibrinogen } \\
\text { depletion }\end{array}$ & Marked & Mild & Moderate & Minimal \\
\hline 90-min patency rates $(\%)$ & $\approx 50$ & $\approx 75$ & $\approx 75$ & $\approx 75$ \\
\hline TIMI grade 3 flow (\%) & 32 & 54 & 60 & 63 \\
\hline
\end{tabular}

\section{Role of statins and potassium channel opener nicorandil in optimizing outcomes in STEMI management:}

Nicorandil and long-acting nitrates are effective drugs used for treatment of chronic stable angina, coronary vasospasm and microvascular angina. Intravenous nicorandil before PCI significantly reduces the frequency of coronary slow flow. Nicorandil also antagonizes ADP induced platelet aggregation, improve microcirculation in ischemia area, decreases no reflow phenomenon and can also inhibit the formation of active oxygen, which is one of the mechanisms in the protective effect on the myocardium. ${ }^{30}$ Although, evidence indicates that treatment with nicorandil is associated with modest reduction in cardiovascular events among patients with stable angina. ${ }^{25}$

Clinical reports have demonstrated various significant effects such as improvement in myocardial perfusion and cardiac function, in TIMI and TMPG score, in STsegment elevation resolution (STR) using nicorandil during PCI in STEMI patients. In addition to this, nicorandil exhibits lower incidence of no-reflow phenomenon (NRP) and was found better tolerated than nitrates without major adverse cardiovascular events.31 Pang et al reported cardioprotective effects of nicorandil in coronary heart disease for, patients undergoing elective PCI by reducing PCI-related MI, rate of the no-reflow phenomenon and improvement of left ventricular ejection fractions. $^{32}$ Recently Feng et al demonstrated improvement in clinical outcomes and decrease in incidence rate of reperfusion injury with early use of nicorandil, distal to vascular lesion during P-PCI in STEMI. $^{33}$ An early coronary use of nicorandil significantly reduced the damage in myocardial microcirculation caused by PPCI, along myocardial infarct size in patients with acute myocardial ischemia. ${ }^{34}$ Reports also suggest that oral nicorandil decreases mortality rates for all patients, especially in elderly patients ( $<75$ years), male gender and patients with hypertension. ${ }^{35}$ Nicorandil is a useful drug for long term outcomes with respect to cardio-protection and ventricular function. A study suggested that oral administration of nicorandil is found associated with reduced incidence of mortality rates and in the setting of secondary prevention. ${ }^{35}$ 
Thereby, combining intravenous thrombolytic agent with oral nicorandil therapy enhances myocardial perfusion level, reduces myocardial damage, improves cardiac function and decreases the risk of arrhythmia. ${ }^{36}$

Statins confer beneficial effects in early secondary prevention and are used as first-line of treatment for ACS patients. ${ }^{37}$ Atorvastatin and rosuvastatin had a favorable effect on lipid profile with a significant decrease in total cholesterol, LDL and triglycerides levels. These two are the most commonly prescribed drugs for hypercholesterolemia and are a part of routine treatment of ACS patients. ${ }^{38}$ Rosuvastatin was found to be more effective in decreasing CRP (C-reactive protein) levels which is associated with greater risk of cardiovascular events. ${ }^{38}$ Rosuvastatin reduces LDL-C, increases HDL-C more effectively and helps in achieving lipid goals than the other statins. Rosuvastatin also demonstrated clinical improvement in atherosclerosis markers such as reduction in lipid rich necrotic core, regression of carotid intimamedia thickness (CIMT), reduction in plaque volume and decreasing apo B/apo A-1ratio. Several clinical studies have also demonstrated that rosuvastatin has a role in regression of coronary atherosclerosis and is beneficial in patients for secondary prevention. ${ }^{39}$ Thus, beyond their lipid-lowering effect, statins are also known to be antiinflammatory, inhibit thrombosis, improve endothelial function, stabilize atherosclerotic plaque, inhibit cell adhesion, and improve microvascular circulation, all of which contribute to improved clinical outcomes in STEMI management. ${ }^{40}$

\section{Strengthening cardiac rehabilitation in India}

Effective follow-up of STEMI patients is crucial for optimal management. In addition to medical interventions, participation in cardiac rehabilitation (CR) program should be strictly recommended to improve patient outcomes after CABG or PCI. Studies have demonstrated that participation in cardiac rehabilitation by patients undergoing myocardial infarction (MI), percutaneous coronary intervention (PCI) and coronary artery bypass graft (CABG) surgery significantly decreases the morbidity, mortality and hospital readmission rates in a cost-effective manner. ${ }^{40,41}$ Cardiovascular associations have recognized the principal CR components which aid in reducing cardiovascular risk, improving quality of life as well as promoting reduced disability. The $\mathrm{CR}$ components includes points such as baseline patient assessment, increasing doctorpatient interaction, imbibing physical activity, training and counselling, nutritional counselling, management of comorbid risk factors e.g. hypertension, dyslipidemia, obesity, diabetes mellitus, etc. as well as psychosocial counselling. ${ }^{42,43}$ Robust clinical evidence have demonstrated that $\mathrm{CR}$ participation reduced morbidity and mortality by $20 \%$ in a cost-effective manner over the subsequent years when compared to usual care. ${ }^{44}$ Thus, efforts should be made to improve cardiac rehabilitation process during hospital stay as well as after hospitalization especially in low-referral hospitals to improve quality of life in patients. ${ }^{45,46}$

In addition to this, hand holding is a crucial approach while creating awareness and educating the healthcare personnel from remote areas. The STEMI India Hub and spoke model (Figure 3) has been reported to be as a reliable model. Replication of a similar program in other towns and cities of India should be encouraged and would ensure appropriate care for STEMI patients. ${ }^{21}$

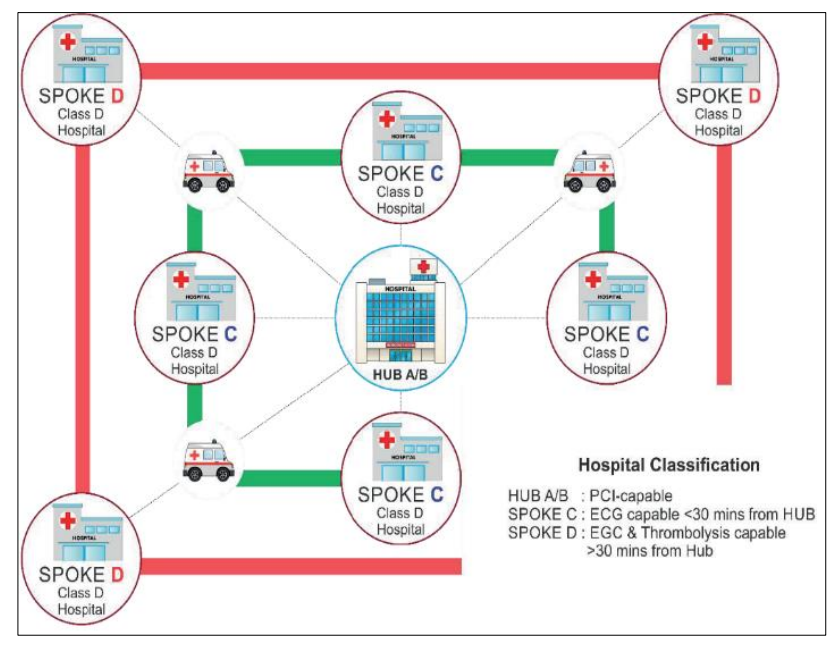

Figure 3: Hub and spoke model of STEMI-India.

\section{CONCLUSION}

Indian metro and tier cities have equipped PCI facility hospitals; however, persistent delays and gaps in timely reperfusion therapy have resulted in increased cardiac mortality rates in India. Pharmaco-invasive therapy can be a critical reperfusion strategic intervention in several patients, where delay in primary percutaneous coronary intervention is anticipated. In rural or urban situations, where there is delay in reaching PCI center, thrombolysis and transfer for PI management is the best option recommended. Administration of nicorandil along with thrombolytic agents was reported to improve cardiac functions. ${ }^{32,36}$ In addition to this, setting up of a cardiac rehabilitation care center is an important crucial step towards improving management goals and long-term survival rates. Extensive work still needs to be conducted in different areas to overcome barriers, which will lead us to a management system beneficial for patients with cardiovascular diseases.

\section{ACKNOWLEDGEMENTS}

The authors would like to thank Scientimed Solutions Pvt Ltd for providing support in manuscript writing.

Funding: No funding sources

Conflict of interest: None declared

Ethical approval: Not required 


\section{REFERENCES}

1. Prabhakaran D, Jeemon P, Roy A. Cardiovascular Diseases in India: Current epidemiology and future directions. Circulation. 2016;133(16):1605-20.

2. Annangi S, Singh T, Maskey A, Gaurav K. Intrathoracic extension of pancreatic pseudo cyst: a rare cause of hemoptysis. Chest. 2019;156(4):A886.

3. Singh A, Grossman SA. Coronary Syndrome, Acute. Stat Pearls. 2017.

4. Hwang C, Levis JT. ECG diagnosis: ST-elevation myocardial infarction. Perm J. 2014;18(2):e133.

5. Alex AG, Lahiri A, Devika. Observational study comparing pharmacoinvasive strategy with primary percutaneous coronary intervention in patients presenting with ST elevation myocardial infarction to a tertiary care centre in India. J Postgrad Med. 2018;64(2):80-5.

6. Ibanez B, James S, Agewall S. 2017 ESC Guidelines for the management of acute myocardial infarction in patients presenting with ST-segment elevation: The Task Force for the management of acute myocardial infarction in patients presenting with ST-segment elevation of the European Society of Cardiology (ESC). Eur Heart J. 2017;39(2):11977.

7. Wald DS, Morris JK, Wald NJ. Randomized trial of preventive angioplasty in myocardial infarction. $\mathrm{N}$ Engl J Med. 2013;369:1115-23.

8. Bueno H, Betriu A, Heras M, Alonso JJ, Cequier A, García EJ, et al. Primary angioplasty vs. fibrinolysis in very old patients with acute myocardial infarction: TRIANA (TRatamiento del InfartoAgudo de miocardioeNAncianos) randomized trial and pooled analysis with previous studies. Eur Heart J. 2010;32(1):51-60

9. Gershlick AH, Khan JN, Kelly DJ, Greenwood JP, Sasikaran T, Curzen N, et al. Randomized trial of complete versus lesion-only revascularization in patients undergoing primary percutaneous coronary intervention for STEMI and multivessel disease: the CvLPRIT trial. J Am Coll Cardiol. 2015;65(10):963-72.

10. Neumann FJ, Sousa-Uva M, Ahlsson A, Alfonso F, Banning AP, Benedetto U, et al. 2018 ESC/EACTS Guidelines on myocardial revascularization. Eur Heart J. 2018.

11. Mohanan PP, Mathew R, Harikrishnan S, Krishnan MN, Zachariah G, Joseph J, et al. Presentation, management, and outcomes of 25748 acute coronary syndrome admissions in Kerala, India: results from the Kerala ACS Registry. Eur Heart J. 2013;34(2):121-9.

12. Xavier D, Pais P, Devereaux PJ, Xie C, Prabhakaran $\mathrm{D}$, Reddy KS, et al. Treatment and outcomes of acute coronary syndromes in India (CREATE): a prospective analysis of registry data. Lancet. 2008;371(9622):1435-42.

13. El Guindy AM. STREAM and FAST-MI Pharmacoinvasive therapy: A continued role for fibrinolysis in the primary PCI era. Glob Cardiol Sci Pract. 2014;2014(2):56-60.

14. Armstrong PW, Gershlick AH, Goldstein P. Fibrinolysis or primary PCI in ST-segment elevation myocardial infarction. $\mathrm{N}$ Engl $\mathrm{J}$ Med. 2013;368(15):1379-87.

15. Danchin N, Puymirat E, Steg PG. Five-year survival in patients with ST-segment-elevation myocardial infarction according to modalities of reperfusion therapy: the French Registry on Acute ST-Elevation and Non-ST-Elevation Myocardial Infarction (FAST-MI) 2005 Cohort. Circulation. 2014;129(16):1629-36.

16. Cahill TJ, Kharbanda RK. Heart failure after myocardial infarction in the era of primary percutaneous coronary intervention: Mechanisms, incidence and identification of patients at risk. World J Cardiol. 2017;9(5):407-15.

17. Meadows ES, Bae JP, Zagar A. Rehospitalization following percutaneous coronary intervention for commercially insured patients with acute coronary syndrome: a retrospective analysis. Brit Med Cent Res Notes. 2012;5:342.

18. Testa L, Van Gaal WJ, Biondi Zoccai GG, Agostoni P, Latini RA, Bedogni F, et al. Myocardial infarction after percutaneous coronary intervention: a meta-analysis of troponin elevation applying the new universal definition. Int $\mathbf{J}$ Medic. 2009; 102(6):369-78.

19. Swieczkowski D, Mogielnicki M, Cwalina N, Zuk G, Pisowodzka I, Ciecwierz D, et al. Medication adherence in patients after percutaneous coronary intervention due to acute myocardial infarction: From research to clinical implications. Cardiol J. 2016;23(5):483-90.

20. Sharma V. Pharmaco-invasive strategy: An attractive alternative for management of STelevation myocardial infarction when timely primary percutaneous coronary intervention is not feasible. J Postgrad Med 2018;64:73-4

21. Alexander T, Juvale NJ, Dasbiswas A, Kubba S, Singh RK, Raja V. Pharmaco-invasive Therapy with Fibrinolytic Agents: A Potent Lifesaving Reperfusion Strategy in STEMI Patients in Metro/Tier-I Cities in India. J Assoc Physic Ind. 2018;66:76.

22. Vos GJ, Bennaghmouch N, Qaderdan K, Ten Berg JM. Management of the patient with an acute coronary syndrome using oral anticoagulation. Netherlands Hea J. 2015;23(9):407-14.

23. Mishra S, Ramakrishnan S, Babu AS. Management algorithms for acute ST elevation myocardial infarction in less industrialized world. Ind Heart J. 2017;69)1):S98-103.

24. Chopra HK. Challenges of STEMI care in India and the real world. Ind Heart J. 2015;67(1):15-7.

25. Tarkin JM, Kaski JC. Nicorandil and Long-acting Nitrates: Vasodilator Therapies for the Management of Chronic Stable Angina Pectoris. Eur Cardiol. 2018;13(1):23-8. 
26. Wander GS, Chhabra ST. Critical analysis of various drugs used for thrombolytic therapy in acute myocardial infarction. Medic Update. 2013;23:10916.

27. Adivitiya, Khasa YP. The evolution of recombinant thrombolytics: Current status and future directions. Bioengineered. 2017;8(4):331-58.

28. Dalal JJ, Alexander T, Banerjee PS, Dayasagar V, Iyengar SS, Kerkar PG, et al. 2013 consensus statement for early reperfusion and pharmacoinvasive approach in patients presenting with chest pain diagnosed as STEMI (ST elevation myocardial infarction) in an Indian setting. J Assoc Physic Ind 2014;62:473.

29. Shah K, Apsangikar P, Allu J. Clinical retrospective and prospective evaluation of efficacy and safety of reteplase in STEMI patients. Ind Med Gaz. 2012;146:479-87.

30. Ye Z, Su Q, Li L. The clinical effect of nicorandil on perioperative myocardial protection in patients undergoing elective PCI: A Systematic Review and Meta-Analysis. Sci Rep. 2017;7:45117.

31. Qi Q, Niu J, Chen T, Yin H, Wang T, Jiang Z. Intracoronary Nicorandil and the Prevention of the No-Reflow Phenomenon During Primary Percutaneous Coronary Intervention in Patients with Acute ST-Segment Elevation Myocardial Infarction. Med Sci Monit. 2018;24:2767-76.

32. Pang Z, Zhao W, Yao Z. Cardioprotective Effects of Nicorandil on Coronary Heart Disease Patients Undergoing Elective Percutaneous Coronary Intervention. Med Sci Monit. 2017;23:2924-30.

33. Feng C, Liu Y, Wang L, Niu D, Han B. Effects of early intracoronary administration of nicorandil during percutaneous coronary intervention in patients with acute myocardial infarction. Hea Lung Circul. 2019;28(6):858-65.

34. Feng C, Han B, Liu Y, Wang L, Niu D, Lou M, et al. Effect of nicorandil administration on myocardial microcirculation during primary percutaneous coronary intervention in patients with acute myocardial infarction. Adva Intervent Cardiol. 2018;14(1):26.

35. Sakata Y, Nakatani D, Shimizu M. Oral treatment with nicorandil at discharge is associated with reduced mortality after acute myocardial infarction. J Cardiol. 2012;59(1):14-21.

36. Yu SH, Li L, Zhang S. Clinical effect of intravenous thrombolysis combined with nicorandil therapy in patients with acute ST-segment elevation myocardial infarction. Tropic J Pharmaceut Res. 2016;15(6):1335-9.
37. Ostadal P. Statins as first-line therapy for acute coronary syndrome? Exp Clin Cardiol. 2012;17(4):227-36.

38. Khurana S, Gupta S, Bhalla H. Comparison of antiinflammatory effect of atorvastatin with rosuvastatin in patients of acute coronary syndrome. J Pharmacol Pharmacotherapeut. 2015;6(3):130.

39. Wander GS, Hukkeri MY, Yalagudri S. Rosuvastatin: Role in Secondary Prevention of Cardiovascular. J Assoc Physic Ind. 2018;66:65-9.

40. Ko YG, Won H, Shin DH. Efficacy of early intensive rosuvastatin therapy in patients with STsegment elevation myocardial infarction undergoing primary percutaneous coronary intervention (ROSEMARY study). Am J Cardiol. 2014;114(1):29-35.

41. Jiménez-Navarro MF, Lopez-Jimenez F, PérezBelmonte LM, et al. Benefits of cardiac rehabilitation on cardiovascular outcomes in patients with diabetes mellitus after percutaneous coronary intervention. J Am Heart Assoc. 2017;6(10):e006404.

42. Kunjan K, Thakur JS, Vijayvergiya R. Effectiveness of cardiac rehabilitation in patients with myocardial infarction and percutaneous coronary intervention at a tertiary care hospital: A pilot intervention study. Int J Non-Commun Dis. 2018;3:104-10.

43. Turk-Adawi K, Sarrafzadegan N, Grace SL. Global availability of cardiac rehabilitation. Nat Rev Cardiol. 2014;11(10):586-96.

44. Grace SL, Turk-Adawi KI, Contractor A. Cardiac rehabilitation delivery model for low-resource settings. Heart. 2016;102(18):1449-55.

45. de MeloGhisi GL, Contractor A, Abhyankar M. Cardiac rehabilitation knowledge, awareness, and practice among cardiologists in India. Indi Hea J. 2018;70(5):753-5.

46. Beatty AL, Bradley SM, Maynard C. Referral to cardiac rehabilitation after percutaneous coronary intervention, coronary artery bypass surgery, and valve surgery: data from the clinical outcomes assessment program. Circulation. 2017;10(6):e003364.

Cite this article as: Dasbiswas A, Shelke A, Singhal A, Shah B. Consensus statement: improvement in care and outcomes for patients with ST-elevation myocardial infarction with timely reperfusion therapy: a pharmaco-invasive approach. Int J Adv Med 2020;7:1889-95. 